\title{
Specification of Timed EFSM Fault Models in SDL
}

\author{
S.S. Batth, E.R. Vieira, A. Cavalli, and M.Ü. Uyar \\ The City College and Graduate Center of the City University of New York, \\ New York, NY 10016, USA \\ \{batth, umit\}@ee-mail.engr.ccny. cuny.edu \\ Laboratoire SAMOVAR (CNRS) and GET/INT Evry Cedex, France \\ \{elisangela.rodrigues, ana.cavalli\}@int-evry.fr
}

\begin{abstract}
In this paper, we apply our timing fault modeling strategy to writing formal specifications for communication protocols. Using the formal language of Specification and Description Language (SDL), we specify the Controller process of rail-road crossing system, a popular benchmark for real-time systems. Our extended finite state machine (EFSM) model has the capability of representing a class of timing faults, which otherwise may not be detected in an IUT. Hit-or-Jump algorithm is applied to the SDL specification based on our EFSM model to generate a test sequence that can detect these timing faults. This application of fault modeling into SDL specification ensures the synchronization among the timing constraints of different processes, and enables generation of portable test sequences since they can be easily represented in other formal notations such as TTCN or MSC.
\end{abstract}

Keywords: Extended Finite State Machines, Timing Fault Models, SDL, Hit-or-Jump.

\section{Introduction}

If the inherent timing constraints are not properly specified in a formal specification of a communication protocol, start and expiration of concurrent timers may lead to infeasible test sequences, which can generate false results by failing correct implementations, or worse, passing the faulty ones.

In this paper, we first introduce an extended finite-state machine (EFSM) model with timer variables based on our earlier work FUDA03, UWBWF05, UBWF06a for the Controller process of the so-called rail-road crossing system ALUR98. This system has been studied as a benchmark in many real-time systems HJL93, HL96, AKLN99, XEN04,CRV05a. We then augment this timed EFSM model such that the test sequences generated from the augmented model, when applied by a tester to an implementation under test (IUT), will detect the presence of a class of timing faults. In this augmentation, a set of new edges and states are created in the system model (i.e., the edge conditions and actions use timing variables as well as the external inputs) such that the resulting model is a 
timed EFSM. In addition, a set of special purpose tester timers are implemented inside the testing system (not in the IUT since the implementation is assumed to be a black box). Only a small number of new states and edges are introduced by our augmentation, and hence the overall length of the test sequences generated from the augmented model, compared to the original system model, does not increase significantly.

We focus on the incorrect timer setting faults EDK02, EDKE98, EKD99, which represent the timers that are incorrectly implemented either too short or too long in Controller. We then provide a formal specification for this system in Specification and Description Language (SDL) [TUZ1, which represents the fault detection capabilities of the augmented EFSM model. In this SDL specification of Controller, a transition of the EFSM fault model that can be triggered when its time constraint is satisfied is represented by one or more continuous signal operators. We specify these EFSM timing constraints by using two variable types in SDL, namely time and duration, which are also used to define the test purposes. To achieve the synchronization among the timing constraints of different processes, we introduce a process, called Clock, to represent the discrete passage of time and use the variable now to verify the global instantiation of the time. The SDL specification can also handle the cases where multiple trains try to cross at the same time.

A test sequence is generated for this SDL specification using the Hit-or-Jump CLRZ99 algorithm. Using the test purposes (also called stop conditions), which represent the timing constraints of the EFSM timing fault model, Hit-or-Jump algorithm constructs efficient test sequences while avoiding the state explosion. In CRV05a, Hit-or-Jump has been applied to railroad crossing system without any fault detection capabilities of our EFSM model. In this paper, we generate the test sequences that are capable of detecting incorrect timer setting faults.

Section 2 of this paper presents an English specification of railroad crossing system. Section 3 introduces the definitions, graph augmentation algorithms GA-A, GA-B and GA-C, and fault modeling for Controller. The SDL specification with timing constraints and test sequence generation using Hit-or-Jump algorithm are in Section 4. The concluding remarks are presented in Section 5.

\section{English Specification for Railroad Crossing System}

The railroad crossing system is one of the popular examples for studying timing constraints in timed FSMs HJL93, HL94, HL96, AKLN99, ALUR98, XEN04. It consists of three main processes: Train, Gate and Controller, all of which must communicate with one another within certain time constraints. Train process communicates with Controller by sending the messages called approach, in, out and exit. The output signal approach must be sent to Controller at least two minutes before a train is crossing the railroad. When a train is inside (or outside) the gate, the corresponding output signal in (or out) is generated. Between the signals approach and exit, there must be a delay of maximum five minutes. When 
Controller receives the input signal approach, it must send the output signal lower to Gate at most one minute after the receipt of approach. If Controller receives exit, it must send the output signal raise to Gate with a maximum delay of one minute.

Gate and Controller communicate through the signals lower, raise, up and down. The signals lower and raise are inputs to Gate process. If lower is received, Gate must respond with down output signal, indicating that the gate is closed and the crossing is safe. The interval between the reception of lower and the sending of down must be at most one minute. If the input signal raise is received by Gate, it must send the output signal up at least one minute and at most two minutes after the receipt of raise.

\section{Modeling Timed Extended Finite State Machines}

A communication protocol modeled as a finite state machine (FSM) can be represented by a directed graph $G(V, E)$. Vertex set $V$ represents the nodes and edge set $E$ represents the edges triggered by events of a system. A protocol specification may include timing variables and operations based their values. To represent these timing related variables, we extend FSMs with timing variables. Our model is complimentary to those presented in timed automata ALUR98, and has the advantage that it is specifically designed for test generation without state explosion [FUDA03].

\subsection{Definitions and Notations}

Let $\mathbf{R}$ denote the set of real, $\mathbf{R}^{\circ+}$ the set of the non-negative real, and $\mathbf{R}^{\infty}=$ $\mathbf{R}^{\circ+} \cup\{-\infty,+\infty\}$ is the set of non-negative real with elements $-\infty$ and $+\infty$. Let $\mathbf{Z}$ denote the set of integers and $\mathbf{Z}^{+}$is the set of positive integers. Interval $[\alpha, \beta]$ is a subset of $\mathbf{R}^{\circ+},[\alpha, \beta] \subset \mathbf{R}^{\circ+}$, and $\delta$ is an instant of $[\alpha, \beta], \delta \in[\alpha, \beta] . \alpha$ is the lower bound of $\delta, \operatorname{Inf}(\delta)=\alpha ; \beta$ is the upper bound of $\delta, \operatorname{Sup}(\delta)=\beta$.

Definition 1. A timed FSM is an FSM augmented to form an Extended Finite State Machine (EFSM), represented by directed graph $G$, denoted by $M=$ $\left(V, A, O, \mathcal{T}, E, v_{0}\right)$ where $V$ is a finite set of nodes, $v_{0} \in V$ is the initial node, $A$ is a finite set of inputs, $O$ is a finite set of outputs, $\mathcal{T}$ is a finite set of variables, and $E$ is a set of edges $V \times A \times \mathcal{T} \longrightarrow V \times O \times \mathcal{T}$. Edge $e_{i} \in E$ can be represented by a tuple $e_{i}=\left(v_{p}, v_{q}, a_{i}, o_{i}, P_{t}(\mathcal{T})=\left\langle e_{i}\right\rangle\right.$, Act $\left._{t}(\mathcal{T})=\left\{e_{i}\right\}\right)$, where $v_{p} \in V$ is a current node, $v_{q} \in V$ is a next node, $a_{i} \in I$ is the input that triggers the transition represented by $v_{p} \stackrel{e_{i}}{\rightarrow} v_{q}, o_{i} \in O$ is the output from current transition $v_{p} \stackrel{e_{i}}{\rightarrow} v_{q}$, $P_{t}(\mathcal{T})=\left\langle e_{i}\right\rangle$ is the set of possible conditions of timing variables. $A_{c} t_{t}(\mathcal{T})=\left\{e_{i}\right\}$ is the set of possible actions on timing variables.

Definition 2. A timer $t m_{j} \in T M$ can be defined with timing variables of $\left(T_{j}, D_{j}, f_{j}\right) \subseteq \mathcal{T}$, where $T M=\left\{t m_{1}, \cdots, t m_{j}, \cdots\right\}$ is a set of $N$ timers, 
$T_{j} \in\{0,1\}$ is a timer running status variable, $D_{j} \in \mathbf{R}^{\circ+}$ is a time-characteristic variable, and $f_{j} \in \mathbf{R}^{\infty}$ is a time-keeping variable.

- Time Keeping Variables $\left(D_{j}\right.$ and $\left.f_{j}\right)$, where $D_{j}$ indicates the length of timer $t m_{j}$, and $f_{j}$ indicates the time elapsed since $t m_{j}$ started. If $t m_{j}$ has just started, $f_{j}:=0$; if $t m_{j}$ is inactive, $f_{j}:=-\infty$. Over an edge $e_{i}$, the value of $f_{j}$ is increased by the amount of time $c_{i} \in \mathbf{R}^{\circ+}$ required to completely traverse the current transition $e_{i}, f_{j}:=f_{j}+c_{i}$. The difference of $\left(D_{j}-f_{j}\right)$ represents the remaining time until $t m_{j}$ 's expiry.

- Timer Status Variable $\left(T_{j}\right)$ is a boolean variable, where $T_{j}==1\left(T_{j}\right)$ denotes timer $t m_{j}$ is active and $T_{j}==0\left(\neg T_{j}\right)$ denotes timer $t m_{j}$ is passive (i.e., stopped, expired or not started yet).

Definition 3. $T M_{\text {active }} \subseteq T M$ and $T M_{\text {passive }} \subseteq T M$ are sets of timers which are active and passive, respectively, such that $T M=T M_{\text {active }} \cup T M_{\text {passive }}$.

- For a transition $e_{i}=\left(v_{p}, v_{q}, a_{i}, o_{i},\left\langle e_{i}\right\rangle,\left\{e_{i}\right\}\right)$, a set of passive timers $t m_{j} \in$ $T M_{\text {passive }}, \forall j \in[1, N]$, can be activated by setting $T_{j}:=1$ and $f_{j}:=0$ in its edge actions. For all the other active timers $t m_{k} \in T M_{\text {active }}, \forall k \in[1, N], k \neq$ $j, f_{k}$ is updated by $e_{i}$ 's traversal time. Formally: $\left\langle e_{i}\right\rangle:\left\langle\neg T_{j} \wedge T_{k} \wedge\left(f_{k}<D_{k}\right)\right\rangle$ and $\left\{e_{i}\right\}:\left\{T_{j}:=1 ; f_{j}:=0 ; T_{k}:=T_{k} ; f_{k}:=f_{k}+c_{i}\right\} \forall k \in[1, N], \forall j \in$ $[1, N], k \neq j$.

- For a transition $e_{i}=\left(v_{p}, v_{q}, a_{i}, o_{i},\left\langle e_{i}\right\rangle,\left\{e_{i}\right\}\right)$, an active timer $t m_{j} \in T M_{\text {active }}$, $j \in[1, N]$, can be stopped by setting $T_{j}:=0$ and $f_{j}:=-\infty$ in its edge actions. For all the other active timers $t m_{k} \in T M_{\text {active }}, \forall k \in[1, N], k \neq j$, $f_{k}$ is updated by $e_{i}$ 's traversal time. Formally: $\left\langle e_{i}\right\rangle:\left\langle T_{j} \wedge\left(f_{j}<D_{j}\right) \wedge T_{k}\right.$ $\left.\wedge\left(f_{k}<D_{k}\right)\right\rangle$ and $\left\{e_{i}\right\}:\left\{T_{j}:=0 ; f_{j}:=-\infty ; T_{k}:=T_{k} ; f_{k}:=f_{k}+c_{i}\right\} \forall k \in$ $[1, N], j \in[1, N], \forall k \neq j$.

- An active timer $t m_{j} \in T M_{\text {active }}$ is defined as expired or timed out iff $f_{j}$ is equal or greater than the timer length $D_{j}$. Formally: $\left\langle T_{j} \wedge\left(f_{j} \geq D_{j}\right)\right\rangle$ and $\left\{T_{j}:=0 ; f_{j}:=-\infty\right\}$.

Definition 4. A transition which becomes feasible when one of the active timers, with the least remaining time, expires is defined as a timeout transition. In other words, $t m_{j} \in T M_{\text {active }}(j \in[1, N]), t m_{k} \in T M_{\text {active }}(\forall k \in[1, N], \forall k \neq j)$, and $t m_{j}$ 's remaining time was the least, then it was $\mathrm{tm}_{j}$ that expired and triggers the timeout edge $e_{i}$. The edge actions set $T_{j}=0, f_{j}=-\infty$, and $f_{k}$ is updated by $e_{i}$ 's traversal time. Formally: $\left\langle e_{i}\right\rangle:\left\langle T_{j} \wedge\left(f_{j} \geqslant D_{j}\right) \wedge T_{k} \wedge\left(D_{j}-f_{j}<D_{k}-f_{k}\right)\right\rangle$ and $\left\{e_{i}\right\}:\left\{T_{j}:=0 ; f_{j}:=-\infty ; T_{k}:=T_{k} ; f_{k}:=f_{k}+c_{i}\right\} \forall k \in[1, N], \forall k \neq j$.

Definition 5. A non-timeout transition becomes feasible iff none of the active timers have expired, or all of the timers are passive. In other words, $\mathrm{tm}_{j} \in$ $T M_{\text {active }}, \forall j \in[1, N]$, and none of these active $t_{j}$ 's have expired. $f_{j}$ is updated by $e_{i}$ 's traversal time. Formally: $\left\langle e_{i}\right\rangle:\left\langle T_{j} \wedge\left(f_{j}<D_{j}\right)\right\rangle$ and $\left\{e_{i}\right\}:\left\{T_{j}:=\right.$ $\left.\left.T_{j} ; f_{j}:=f_{j}+c_{i}\right)\right\} \forall j \in[1, N]$. 
Definition 6. Flow Enforcing Variable $\left(L_{p}\right)$ is an exit condition to leave a state $v_{p}$. It is denoted by a boolean variable $L_{p} \in\{0,1\} \forall v_{p} \in V$, where $L_{p}==0$ means none of the transitions is allowed to leave $v_{p}$, and $L_{p}==1$ means transitions are allowed to leave $v_{p}$.

Definition 7. A transition whose action updates $L_{p}$ from 0 to 1 is defined as an observer edge. Formally: $\left\langle e_{p, o b s}\right\rangle:\left\langle L_{p}==0\right\rangle$ and $\left\{e_{p, o b s}\right\}:\left\{L_{p}:=1\right\} \forall v_{p} \in V$.

Definition 8. For an active timer, a transition which consumes the pending timeout is defined as a wait edge. In other words, $t m_{j} \in T M_{\text {active }}(j \in[1, N])$, $t m_{k} \in T M_{\text {active }}(\forall k \in[1, N], \forall k \neq j)$ and $t m_{j}$ 's remaining time is the least, then the wait edge updates $f_{j}$ by $\mathrm{tm}_{j}$ 's remaining time $D_{j}-f_{j}$. Formally: $\left\langle e_{p, \text { wait }}\right\rangle$ : $\left\langle T_{j} \wedge\left(f_{j}<D_{j}\right) \wedge T_{k} \wedge\left(f_{k}<D_{k}\right) \wedge\left(D_{j}-f_{j}<D_{k}-f_{k}\right)\right\rangle$ and $\left\{e_{p, \text { wait }}\right\}:\left\{f_{j}:=\right.$ $\left.f_{j}+\left(D_{j}-f_{j}\right) ; f_{k}:=f_{k}+\left(D_{j}-f_{j}\right)\right\} \forall k \neq j, k \in[1, N], \forall v_{p} \in V$.

Definition 9. A return edge is an edge with no time constraints and actions: $\left\langle e_{p}^{r e t}\right\rangle:\langle 1\rangle$ and $\left\{e_{p}^{r e t}\right\}:\{\} \forall v_{p} \in V$.

Definition 10. During testing an edge $e_{i}=\left(v_{p}, v_{q}, a_{i}, o_{i},\left\langle\mathbf{e}_{i}\right\rangle,\left\{\mathbf{e}_{i}\right\}\right)$, after input $a_{i}$ is applied to an IUT, the expected output $o_{i}$ should be generated no later than a certain $\theta$ time units, $\theta \in \mathbf{R}^{\circ+}$, measured by a timer which is a part of the test harness rather than the IUT.

\subsection{Graph Augmentation Algorithm GA-A}

To model the original system along with its timed behavior, we introduce a graph augmentation algorithm, called GA-A UBWF06a], which is specifically designed for generating tests for the systems whose timer related variables are linear and their values implicitly increase with time. To ensure that the timing conditions and actions of the specification are correctly incorporated into the timed EFSM model, GA-A generates $G^{\prime}\left(V^{\prime}, E^{\prime}\right)$ by converting self-loops in $G$ to node-to-node edges, defining an exit condition for all the nodes, and creating a set of new nodes and edges:

Step (i): If there exists a self loop for $v_{p} \in V$ in $G$, an additional node called $v_{p}^{\prime}$ is created in $G^{\prime}$, to which all self-loops $e_{p, k} \in E$ defined in $v_{p}$ are directed;

Step (ii): All self-loops $e_{p, k} \in E$ in $G$ are converted to node-to-node edges in $G^{\prime}$ as $e_{p, k}=\left(v_{p}, v_{p}^{\prime}\right)$.

Step (iii): For $v_{p}^{\prime} \in V^{\prime}$ in $G^{\prime}$, a return edge $e_{p}^{r e t}$ from $v_{p}^{\prime}$ to $v_{p}$ is created in $G^{\prime}$ as $e_{p}^{r e t}=\left(v_{p}^{\prime}, v_{p}\right)$.

Step (iv): An observer node is created in $G^{\prime}$, namely $v_{p, \text { wait }}$, which is connected to $v_{p}$ via newly created an observer edge as $e_{p, o b s}=\left(v_{p}, v_{p, \text { wait }}\right)$, a wait edge as $e_{p, \text { wait }}=\left(v_{p}, v_{p, \text { wait }}\right)$, and a return edge from observer node as $e_{p, o b s}^{r e t}=\left(v_{p, w a i t}, v_{p}\right)$. The role of the observer node $v_{p, \text { wait }}$ is to consume pending timeouts on $e_{p, w a i t}$ and enable outgoing edges by setting the flow enforcing variable $L_{p}$ to 1 on $e_{p, o b s}$. Fig. 1 1 shows, for node $v_{p}$, the conversion of self-loops to node-to-node edges, the creation of the observer node, wait and observer edges. 


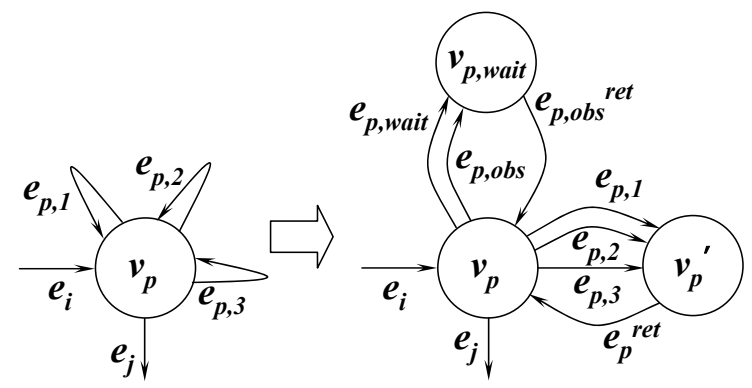

Fig. 1. Modeling self-loops for $v_{p}$ in $\mathrm{G}$ into $v_{p}, v_{p}^{\prime}$ and $v_{p, \text { wait }}$ in $G^{\prime}$

The time condition and the action for the wait edge $e_{p, w a i t}$ are formulated as $\left\langle L_{p}==0\right\rangle$ and $\left\{f_{j}:=f_{j}+\left(D_{j}-f_{j}\right)\right\}$, where $D_{j}-f_{j}$ is the remaining time of timer $t m_{j} \in T M_{\text {active }}$ to timeout. For the observer edge $e_{p, o b s}$ from the original node $v_{p}$ to the observer node $v_{p, \text { wait }}$ in $G^{\prime}$, the time condition and the action are formulated as $\left\langle L_{p}==0\right\rangle$ and $\left\{L_{p}:=1\right\}$, respectively. The return edges of $e_{p}^{r e t}$ and $e_{p, o b s}^{r e t}$ are added by GA-A to $G^{\prime}$ are no-cost edges with time condition as: $\langle 1\rangle$ (i.e., always true with no time constraints imposed) with no actions: \{\} .

Step $(v)$ : The conditions and actions for a timeout edge in $G^{\prime}$ are:

- The condition for a timeout self-loop edge in $G$ becomes: $\left\langle T_{j} \wedge\left(f_{j} \geqslant D_{j}\right) \wedge\right.$ $\left.T_{k} \wedge\left(f_{k}<D_{k}\right) \wedge\left(D_{j}-f_{j}<D_{k}-f_{k}\right) \wedge\left(L_{p}==0\right)\right\rangle \forall T_{k} \neq T_{j}$, where the remaining time for $t m_{j} \in T M_{\text {active }}$ is less than that of $t m_{k} \in T M_{\text {active }}$ (i.e., $D_{j}-f_{j}<D_{k}-f_{k}$ ) and the flow enforcing variable $L_{p}$ is zero.

- The condition for a timeout node-to-node edge in $G$ becomes: $\left\langle T_{j} \wedge\left(f_{j} \geqslant\right.\right.$ $\left.\left.D_{j}\right) \wedge T_{k} \wedge\left(f_{k}<D_{k}\right) \wedge\left(D_{j}-f_{j}<D_{k}-f_{k}\right) \wedge\left(L_{p}==1\right)\right\rangle \forall T_{k} \neq T_{j}$, where the remaining time for $t m_{j} \in T M_{\text {active }}$ is less than that of $t m_{k} \in T M_{\text {active }}$ (i.e., $\left.D_{j}-f_{j}<D_{k}-f_{k}\right)$ and $L_{p}$ is 1 .

- The actions for a timeout edge in $G$ become: $\left\{T_{j}:=0 ; f_{j}:=-\infty ; T_{k}:=\right.$ $\left.T_{k} ; f_{k}:=f_{k}+c_{i} ; L_{p}:=0\right\} \forall t m_{k} \neq t m_{j}$, where timer $t m_{j} \in T M_{\text {passive }}$ becomes passive and the time keeping variable for $t m_{k} \in T M_{\text {active }}$ is incremented by the edge cost of $c_{i}$.

These equations imply that before a timeout edge, $t m_{j}$ should be still running, remaining time should be the least among all other running timers and the flowenforcing variable is appropriately set for either a converted (i.e., self-loop edge in $G$ ) or an original (i.e., node to node edge in $G$ ) edge in $G^{\prime}$.

Step (vi): The conditions and actions for a non-timeout edge in $G^{\prime}$ is formalized as follows:

- A non-timeout self-loop edge in $G$ becomes: $\left\langle\left(\neg T_{j} \vee\left(T_{j} \wedge\left(f_{j}<D_{j}\right)\right)\right) \wedge\left(L_{p}==\right.\right.$ 0) $\forall \forall t m_{j} \in T M_{\text {active }}$

- A non-timeout node-to-node edge in $G$ becomes: $\left\langle\left(\neg T_{j} \vee\left(T_{j} \wedge\left(f_{j}<D_{j}\right)\right)\right) \wedge\right.$ $\left.\left(L_{p}==1\right)\right\rangle \forall t m_{j} \in T M_{\text {active }}$

- The action for a non-timeout edge in $G$ becomes: 
- $\left\{f_{j}:=f_{j}+c_{i} ; f_{k}:=f_{k}+c_{i} ; L_{p}:=0\right\} \forall t m_{k} \neq t m_{j}, t m_{j} \in T M_{\text {active }}, t m_{k} \in$ $T M_{\text {active }}$ if edge starts no timers;

- $\left\{T_{j}:=1 ; f_{j}:=0 ; T_{k}:=T_{k} ; f_{k}:=f_{k}+c_{i} ; L_{p}:=0\right\} \forall t m_{k} \neq t m_{j}$ if edge starts timer $\mathrm{tm}_{j}$.

Since both timeout and non-timeout edges disable outgoing edges by setting $L_{p}:=0$ in Steps $(v)$ and (vi) of GA-A, the only edges whose actions will enable the outgoing edges in $G^{\prime}$ are the artificially-created observer edges.

It is proven UBWF06a that GA-A terminates with a running time of $\mathbb{O}(E)$, and that the total number of the nodes and edges in $G^{\prime}\left(V^{\prime}, E^{\prime}\right)$ and $G(V, E)$ have the same order of magnitude.

\subsection{Classification of Timing Faults}

A class of timing faults in an implementation of a timed system have been defined in EDK02,EDKE98,EKD99 as 1-clock timing faults (including 1-clock corner point and 1-clock interval faults) and incorrect timer length setting faults.

Incorrect Timer Setting Faults occur in an IUT when a timer length is incorrectly implemented as either too short or too long (i.e., the timer expires either too early or too late). The definition of incorrect timer setting faults is based on the following timing requirement:

- Timing Requirement: In a test sequence, edge $h_{k}$ starts timer $t m_{j}$ and is traversed before $e_{i}$. Timeout transition $e_{i}=\left(v_{p}, v_{q}\right.$, timeout_tm $_{j}, o_{i},\left\langle\mathbf{t}_{j}\right\rangle$, $\left.\left\{\mathbf{t}_{j}\right\}\right)$ triggers exactly in $D_{j}$ time units, where $D_{j}$ is the timer length.

- Timing Fault $\boldsymbol{B}\left(T F_{B}\right)$ : Timeout transition $e_{i}$ triggers in $D_{j}^{\prime}$ time units and output $o_{i}$ is observed and node $v_{q}$ is verified in shorter than the expected time (i.e., $D_{j}^{\prime}<D_{j}$ ).

- Timing Fault $\boldsymbol{C}\left(T F_{C}\right)$ : Timeout transition $e_{i}$ triggers in $D_{j}^{\prime}$ time units and output $o_{i}$ is observed and node $v_{q}$ is verified in longer than the expected time (i.e., $D_{j}^{\prime}>D_{j}$ ).

In a specification, suppose a timer $t m_{j}$ is defined to be of length $D_{j}$ to be started by the actions of edge $h_{k}$ and to expire at edge $e_{i}$ (reachable from $h_{k}$ ). A special purpose timer $t m_{s}$ with length $D_{s}=D_{j}$ is created in the test harness by GA-2. B to detect if $t m_{j}$ is set too short as $D_{j}^{\prime}<D_{j}$ :

Step (B.i): Edge conditions and actions for $h_{k}$ are modified such that it starts a special purpose timer $t m_{s}$.

Step (B.ii): $e_{i}$ 's condition is modified such that it traverses only when both $t m_{s}$ and $t m_{j}$ have expired.

Step (B.iii): All self-loops in $v_{p}$ are represented as node-to-node edges by the creation of an additional node, called $v_{p}^{\prime}$, to which they are directed. A return edge $e_{p}^{r e t}$ (with zero cost) is also created for their return to $v_{p}$.

Step (B.iv): An observer node $v_{p, \text { wait }}$ is appended to node $v_{p}$ via a new observer edge $e_{p, o b s}$, wait edge $e_{p, \text { wait }}$ (with cost $c_{p, \text { wait }}$ ) and return edge $e_{p}^{r e t}$ (with cost $c_{p}^{r e t}:=0$ ). The edge condition of $e_{i}$ is modified such that it triggers only when $f_{s} \geqslant D_{s}$ and $t m_{j}$ expires. 
As proven in UBWF06a, GA-2.B terminates with a running time of $\mathbb{O}(E)$, and the order of magnitude of the nodes and edges in $G^{\prime}$ and $G^{\prime \prime}$ are the same. A test sequence generated from $G^{\prime \prime}$ will contain $\cdots, h_{k}, \cdots, e_{i-1}, e_{p, \text { wait }}, e_{p}^{r e t}$, $e_{p, o b s}, e_{p}^{r e t}, e_{i}$ which will not be feasible to traverse if timer $t m_{j}$ expires earlier than expected. The condition for $e_{p \text {,wait }}$ requires that both the timers $t m_{j}$ from the IUT and $t m_{s}$ from the test harness are still running. If $t m_{j}$ times out before $t m_{s}$, it will create a deadlock at $v_{p}$ (i.e., none of the conditions leaving $v_{p}$ is valid), which in turn will flag the tester that a timing fault $T F_{B}$ has occurred.

Algorithm GA-2.C [UBWF06a] for $T F_{C}$, is similar to GA-2.B, with the same run time complexity and the augmented graph size of $G^{\prime}$.

\subsection{Timed EFSM Model for Railroad Crossing System}

Due to space constraints, we only consider timing fault $T F_{B}$ in the edges of $e_{2}$ and $e_{4}$ in Controller, whose FSM model is given in Fig. 2 The steps for generating graph $G^{\prime \prime}$ is follows:

Step 1: Obtain graph $G$ from the specification of Controller process. The directed graph representing Controller is in Fig. 2 with its actions and conditions given in Table 1. Timer $t m_{z}$ can be started either in edge $e_{1}$ or in $e_{3}$ with the timer length of 1 min (i.e., $D_{z}=1 \mathrm{~min}$ ).

Step 2: Generate $G^{\prime}$ for Controller by applying the graph augmentation algorithm GA-A to $G$. The new observer nodes and edges (i.e., $s_{0, \text { wait }}, e_{0, \text { wait }}$, $e_{0, o b s}, e_{0, o b s}^{r e t}, s_{1, w a i t}, e_{1, w a i t}, e_{1, o b s}, e_{1, o b s}^{r e t}, s_{2, w a i t}, e_{2, w a i t}, e_{2, o b s}, e_{2, o b s}^{r e t}, s_{3, w a i t}$, $\left.e_{3, \text { wait }}, e_{3, o b s}, e_{3, o b s}^{r e t}\right)$ are added to the original nodes of $G$. The self-loop edge of $e_{0}$ is converted to a node-to-node edge by introducing $s_{0}^{\prime}$ and $e_{0}^{r e t}$ in $G^{\prime}$.

Step 3: Apply the graph augmentation algorithm GA-B to $G^{\prime}$ to generate $G^{\prime \prime}$ for Controller. A special purpose timer, namely $t m_{s}$ (with $D_{s}=1$ ), is introduced in the tester (not in the IUT) to model the timing constraints over the edges of $e_{2}$ and $e_{4}$. Note that, in $G^{\prime \prime}, e_{1}$ starts both the special purpose timer $t m_{s}$ in the tester and the timer $t m_{z}$ in the IUT; similarly, $e_{3}$ starts the same two timers in the tester and the IUT. Graph $G^{\prime \prime}$ is shown in Fig. 3 with its respective edge conditions and actions given in Table 2.

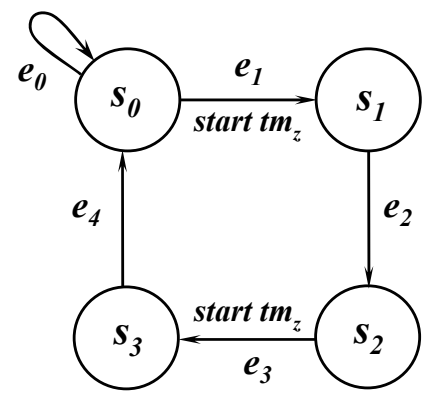

Fig. 2. Finite state machine for Controller 
Table 1. Original specification of Controller (Fig. 2) and its graph $G$

\begin{tabular}{|c|c|c|c|}
\hline Edges & English Specification & \multicolumn{2}{|c|}{ Our EFSM Model $G$} \\
\cline { 3 - 4 } & & $\begin{array}{c}\text { Timing } \\
\text { Conditions }\end{array}$ & $\begin{array}{c}\text { Timing } \\
\text { Actions }\end{array}$ \\
\hline$e_{0}$ & Idle & $\langle 1\rangle$ & \{\} \\
\hline$e_{1}$ & Input approach is received & $\left\langle\left(a_{1}==\right.\right.$ approach $\left.)\right\rangle$ & $\left\{T_{z}:=1 ; f_{z}:=0\right\}$ \\
\hline$e_{2}$ & $\begin{array}{c}\text { Output lower is generated at } \\
\text { maximum delay of } 1 \text { mins after } \\
\text { input approach is received }\end{array}$ & $\left\langle T_{z} \wedge\left(f_{z} \geq D_{z}\right)\right\rangle$ & $\left\{o_{2}:=\right.$ lower $;$ \\
$\left.T_{z}:=0 ; f_{z}:=-\infty\right\}$ \\
\hline$e_{3}$ & Input exit is received & $\left\langle\left(a_{3}==\right.\right.$ exit $\left.)\right\rangle$ & $\left\{T_{z}:=1 ; f_{z}:=0\right\}$ \\
\hline$e_{4}$ & $\begin{array}{c}\text { Output raise is generated } \\
\text { maximum delay of } 1 \text { mins } \\
\text { after input exit is received }\end{array}$ & $\left\langle T_{z} \wedge\left(f_{z} \geq D_{z}\right)\right\rangle$ & $\left\{o_{4}:=\right.$ raise; \\
$\left.T_{z}:=0 ; f_{z}:=-\infty\right\}$ \\
\hline
\end{tabular}

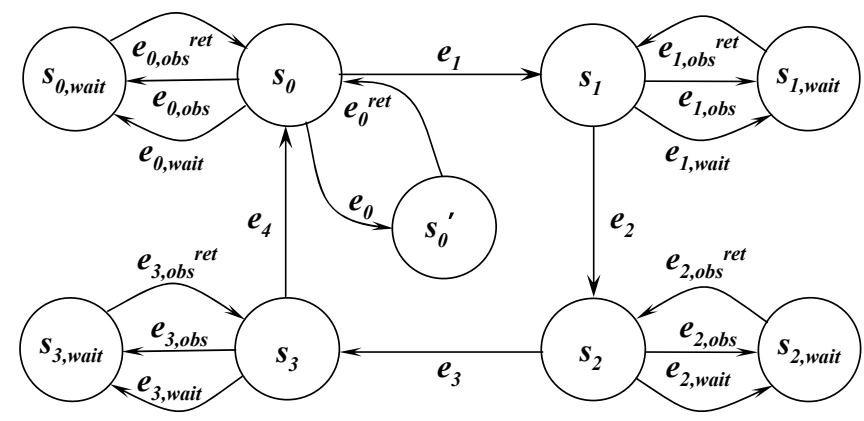

Fig. 3. Augmented Graph $G^{\prime \prime}$ for Controller (Fig. 2) after applying GA-A and GA-B

\section{SDL Specification Based on Timed EFSM Model}

To specify a set of timed EFSM models in SDL one may either (i) define each component (e.g., Train, Gate and Controller) as an independent system, where each exchange messages with the environment, or (ii) define each component as a process of the same system. Although both approaches are equivalent, in this paper we follow the latter approach. Our SDL specification is designed for testing purposes, where the evolution of time is modeled by the expiration of the clocks. We introduce a process, called Clock, as a part of the Railroad system to represent the passage of time. Therefore, our SDL specification for the railroad crossing system consists of a main Railroad system, which includes a Railroad_Control block (Fig. 4) with four processes, namely Train, Gate, Controller and Clock.

In our EFSM model, each edge $e_{i}$ is associated with a timing cost $c_{i}$, representing the expected time that is required to traverse (or, realize) the edge in an implementation (see Section 3). The corresponding state transition in SDL 
Table 2. Augmented edge conditions and actions of graph $G^{\prime \prime}$ (Fig. 3) of Controller

\begin{tabular}{|c|c|c|}
\hline Edges & $\langle$ Edge Conditions $\rangle$ & $\{$ Edge Actions $\}$ \\
\hline$e_{0}$ & $\langle\neg$ approach $\rangle$ & \{\} \\
\hline$e_{0}^{\text {ret }}$ & $\langle 1\rangle$ & \{\} \\
\hline$e_{0, o b s}$ & $\left\langle L_{p}==0\right\rangle$ & $\left\{L_{p}:=1\right\}$ \\
\hline$e_{0, \text { wait }}$ & $\left\langle\neg\right.$ approach $\left.\wedge L_{p}==0\right\rangle$ & $\left\{f_{i}:=f_{i}+c_{0, w a i t}\right\}$ \\
\hline$e_{0, o b s}^{r e t}$ & $\langle 1\rangle$ & \{\} \\
\hline$e_{1}$ & $\left\langle\right.$ approach $\left.\wedge L_{p}==1\right\rangle$ & $\left\{T_{s}:=1 ; f_{s}:=0 ; L_{p}:=0\right\}$ \\
\hline$e_{1, \text { wait }}$ & $\left\langle L_{p}==0\right\rangle$ & $\left\{f_{i}:=f_{i}+c_{1, \text { wait }}\right\}$ \\
\hline$e_{1, o b s}$ & $\left\langle L_{p}==0\right\rangle$ & $\left\{L_{p}:=1\right\}$ \\
\hline$e_{1, o b s}^{r e t}$ & $\langle 1\rangle$ & \{\} \\
\hline$e_{2}$ & $\left\langle T_{s} \wedge\left(f_{s} \geq D_{s}\right) \wedge\left(T_{z}\right.\right.$ timeout $\left.) \wedge L_{p}==1\right\rangle$ & $\left\{\right.$ lower $\left.; T_{s}:=0 ; f_{s}:=-\infty ; L_{p}:=0\right\}$ \\
\hline$e_{2, \text { wait }}$ & $\left\langle\neg\right.$ exit $\left.\wedge L_{p}==0\right\rangle$ & $\left\{f_{i}:=f_{i}+c_{2, \text { wait }}\right\}$ \\
\hline$e_{2, o b s}$ & $\left\langle L_{p}==0\right\rangle$ & $\left\{L_{p}:=1\right\}$ \\
\hline$e_{2, o b s}^{r e t}$ & $\langle 1\rangle$ & \{\} \\
\hline$e_{3}$ & $\left\langle\right.$ exit $\left.\wedge L_{p}==1\right\rangle$ & $\left.T_{s}:=1 ; f_{s}:=0 ; L_{p}:=0\right\}$ \\
\hline$e_{3, \text { wait }}$ & $\left\langle L_{p}==0\right\rangle$ & $\left\{f_{i}:=f_{i}+c_{3, \text { wait }}\right\}$ \\
\hline$e_{3, o b s}$ & $\left\langle L_{p}==0\right\rangle$ & $\left\{L_{p}:=1\right\}$ \\
\hline$e_{3, o b s}^{r e t}$ & $\langle 1\rangle$ & \{\} \\
\hline$e_{4}$ & $\left\langle T_{s} \wedge\left(f_{s} \geq D_{s}\right) \wedge\left(T_{z}\right.\right.$ timeout $\left.) \wedge L_{p}==1\right\rangle$ & $\left\{\right.$ raise $\left.; T_{s}:=0 ; f_{s}:=-\infty ; L_{p}:=0\right\}$ \\
\hline
\end{tabular}

specification can be represented as the difference between two internal variables that are set at the instances of the beginning and end of the transition. This way, these two variables, one with the clock value at the beginning and the other one at the end, can be used to approximate the edge traversal time in SDL, Similarly, the following assumptions are considered to specify a real-time system in SDL AKLN99, TMCB03:

- All un-timed events will take a negligible time to realize;

- Time advances through the expiration of local clocks; if two clocks expire at the same moment, only one of them is taken into account first;

- As time progresses, time dependent transitions may trigger only if their conditions are satisfied;

- The global clock called now is the only clock which gives the current time.

In this approach, time constraints are represented as continuous signal operators. This construct allows to represent a transition that does not need an input signal to be fired, but is triggered when the time constraint is satisfied. In our SDL specification, two variable types are used: a time variable to register the moment when an event occurs, and a duration variable to represent the difference between two time variables. For example, in the timing condition of $\left(f_{1}-f_{2}>D_{2}\right)$, variables $f_{1}$ and $f_{2}$ are of type time, whereas $D_{2}$ is a duration variable. Both time and duration variables are also defined in our EFSM model in Section 3. For example, for the special purpose timer $t m_{s}$ in $G^{\prime \prime}$ (Section 3.3), time keeping variable $f_{s}$ and the timer length $D_{s}$ are represented as the time and duration types of variables in our SDL specification, respectively. 


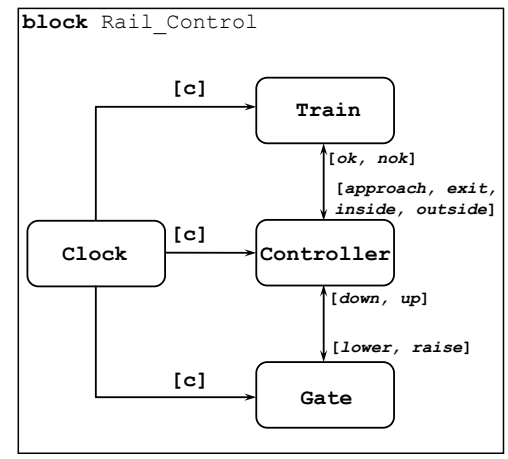

Fig. 4. Rail_Control block of SDL specification

Although we did not utilize the local timer construct in SDL to represent the timer $t m_{s}$, we have instead used the variable now and the process Clock to model the evolution of time in our SDL specification. Therefore, for Controller, $f_{s}$ is represented by four time type variables, namely zapproach, zexit, zlower and zraise. The moment when approach and exit signals are received is represented by zapproach and zexit, respectively. Similarly, zlower and zraise are used to capture the moment when lower and raise are sent, respectively. Timer length $D_{s}$ is modeled by two duration type variables, namely sent_lower_delay and sent_raise_delay, both equal to $1 \mathrm{~min}$. Table 3 illustrates the relationship between our SDL specification and the EFSM model based on $G^{\prime \prime}$.

Our SDL specification also allows representation of more than one train trying to cross at the same time. To model multiple trains, additional variables such as (ntrains and max_trains), and signals ( ok and nok) are introduced (in the SDL specification given in this paper, max_trains $=1)$. Since there are a limited number of tracks available, variable ntrains counts the number of trains which have sent approach to Controller. Each approach received from a different train can be distinguished by Controller because an internal identifier with a distinct channel is created for each instantiation of the Train process. Therefore, if the condition of (ntrains < = max_trains) is true, Controller sends ok; otherwise it sends nok. If Train receives ok from Controller, the train continues its approach to the railroad crossing. Similarly, if nok is received by Controller, the train waits until it receives a signal of $o k$. When one of the Train processes sends exit, Controller decrements the value of ntrains by one. If the updated value of ntrains is still greater than zero, Controller sends another ok to one of the Train processes waiting to approach the railroad crossing; otherwise, Controller sends raise signal to Gate.

\subsection{Application of Hit-or-Jump Algorithm}

Hit-or-Jump CLRZ99] algorithm can be used for embedded testing of complex communication systems which are modeled as communicating EFSMs. It is a generalization and unification of exhaustive search and random walks; both of which 


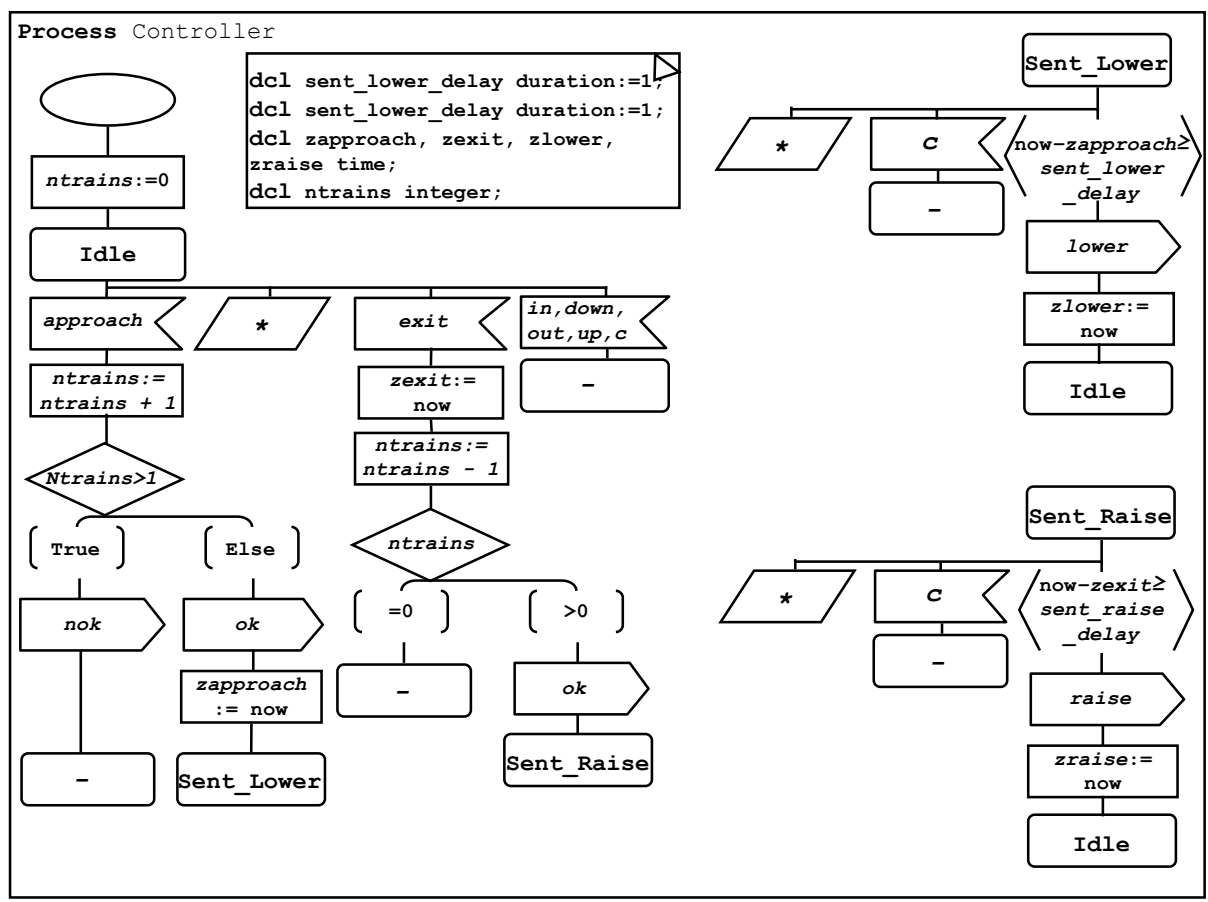

Fig. 5. SDL specification of Controller

are special cases of Hit-or-Jump. It efficiently constructs testing sequences with a high fault coverage, does not suffer from the drawback of state space explosion as encountered in exhaustive search, and quickly covers the system components under test without being trapped, as experienced by random walks. Furthermore, it has also been applied to embedded testing of telephone services CLRZ99, conformance and interoperability testing of web services CMZ04 and in the domain of real-time systems CRV05a, CRV05b. The strategy used to generate a partial accessibility graph in Hit-or-Jump is that if a visited node satisfies the test purposes, it is said that a hit is done; otherwise, the algorithm randomly choses another node from the neighborhood graph, and moves (jump) to it. Then from this new node, it continues its search. Parameters required to execute the Hit-or-Jump are:

(i) SDL specification of the IUT (Fig. 5);

(ii) Test purposes described in several stop conditions, which are the properties to be verified at each node. Each property can be defined in input signals, output signals, time, and duration variable types. In our case study, the test purposes are defined according to the timing fault models of $G^{\prime \prime}$ graph. These are then modeled for SDL specification and used as stop conditions. Table 4 gives the details of test purposes for all the processes of the railroad crossing system; 
Table 3. Relationship between SDL Specification (Fig 5) and EFSM Model (Fig 3) for Controller

\begin{tabular}{|c|c|c|c|c|c|c|}
\hline \multicolumn{2}{|c|}{ Current State } & \multicolumn{2}{|c|}{ Next State } & \multirow{3}{*}{\begin{tabular}{|c|} 
Edge \\
Name
\end{tabular}} & \multirow[t]{3}{*}{ Constraint } & \multirow[t]{3}{*}{ Action } \\
\hline SDL & EFSM & SDL & EFSM & & & \\
\hline Spec. & Model & Spec. & Model & & & \\
\hline Start & & Idle & $s_{0}$ & & & ntrains $:=0$ \\
\hline Idle & $s_{0}$ & Idle & $s_{0}$ & & $\begin{array}{c}\text { (approach?) and } \\
(\text { ntrains }>1)\end{array}$ & $\begin{array}{c}(\text { nok!) and } \\
(\text { zapproach:= now) }\end{array}$ \\
\hline Idle & $s_{0}$ & Sent_Lower & $s_{1}$ & $e_{1}$ & $\begin{array}{c}\text { (approach?) and } \\
(\text { ntrains } \leqslant 1)\end{array}$ & $\begin{array}{c}(o k !) \text { and } \\
(\text { zapproach:= now) }\end{array}$ \\
\hline Sent_Lower & $s_{1}$ & Sent_Lower & $s_{1, \text { wait }}$ & $e_{1, \text { wait }}$ & $\begin{array}{l}\quad(* ?) \text { or } \\
\text { (now }- \text { zapproach } \leqslant \\
\text { sent_lower_delay) }\end{array}$ & \\
\hline Sent_Lower & $s_{1}$ & Idle & $s_{2}$ & $e_{2}$ & $\begin{array}{c}\text { (now }- \text { zapproach } \geqslant \\
\text { sent_lower_delay) }\end{array}$ & $\begin{array}{c}\text { (lower!) and } \\
\text { (zlower := now) }\end{array}$ \\
\hline Idle & $s_{2}$ & Idle & $s_{2}$ & & $\begin{array}{c}(\text { exit } ?) \text { and } \\
(\text { ntrains } \geqslant 0)\end{array}$ & $\begin{array}{c}(o k !) \text { and } \\
\text { (zexit }:=\text { now) }\end{array}$ \\
\hline Idle & $s_{2}$ & Sent_Raise & $s_{3}$ & $e_{3}$ & $\begin{array}{c}(\text { exit }) \text { and } \\
(\text { ntrains } \leqslant 0)\end{array}$ & (zexit := now) \\
\hline Sent_Raise & $s_{3}$ & Sent_Raise & $s_{3}$ & $e_{3, \text { wait }}$ & $\begin{array}{c}\quad(* ?) \text { or } \\
\text { (now }- \text { zexit } \leqslant \\
\text { sent_raise_delay) }\end{array}$ & \\
\hline Sent_Raise & $s_{3}$ & Idle & $s_{0}$ & $e_{4}$ & $($ now - zexit $\geq 1)$ & $\begin{array}{c}\text { (raise!) and } \\
\text { (zraise }:=\text { now) }\end{array}$ \\
\hline \multicolumn{7}{|c|}{$\begin{array}{l}\text { Legend: Input }=? \text {, Output }=\text { !, now }=\text { Global Clock, } *=\text { Any other signal; } \\
\text { Time type variables = zapproach, zexit, zraise, zlower; } \\
\text { Duration type variables = sent_lower_delay, sent_raise_delay }\end{array}$} \\
\hline
\end{tabular}

Table 4. Test purposes for SDL specification and EFSM model

\begin{tabular}{|c|c|c|}
\hline $\begin{array}{c}\text { Process } \\
\text { Name }\end{array}$ & $\begin{array}{c}\text { Test Purposes for } \\
\text { EFSM Model }\end{array}$ & $\begin{array}{c}\text { Test Purposes for } \\
\text { SDL Specification }\end{array}$ \\
\hline Train & $\begin{array}{c}\text { Output } \text { in is generated in less } \\
\text { than 2 minutes after approach }\end{array}$ & xinside - xapproach $<2$ \\
\cline { 2 - 3 } & $\begin{array}{c}\text { Output exit is generated in more } \\
\text { than 5 minutes after approach }\end{array}$ & xexit - xapproach $>5$ \\
\hline Controller & $\begin{array}{c}\text { Output lower is generated in less } \\
\text { than } 1 \text { minutes after approach }\end{array}$ & zlower - zapproach $<1$ \\
\cline { 2 - 3 } & $\begin{array}{c}\text { Output raise is generated in more } \\
\text { than } 1 \text { minutes after } \text { exit }\end{array}$ & zraise - zexit $>1$ \\
\hline Gate & $\begin{array}{c}\text { Output down is generated in more } \\
\text { than } 1 \text { minutes after lower }\end{array}$ & ydown - ylower $>1$ \\
\cline { 2 - 3 } & $\begin{array}{c}\text { Output up is generated in more } \\
\text { than } 2 \text { minutes after raise }\end{array}$ & yup - yraise $>2$ \\
\hline
\end{tabular}

(iii) A preamble scenario (optional) may be furnished in order to guide the algorithm to easily and quickly find a sequence which satisfies the stop 
conditions (test purposes). If no preamble scenario is given, the search starts from the initial state of all processes;

(iv) The strategy of the search, which can either be a breadth or a depth search, in order to generate an internal accessibility graph;

(v) A local search parameter (an integer), which defines the space required for the search before a jump.

The test sequence generated from SDL specification of Controller by applying Hit-or-Jump is given in Table 5. Note that all un-timed transitions have zero cost because of the assumption in SDL that these transitions take insignificant time to run. The cost of the wait edges is expressed in minutes.

Using our SDL specification, Hit-or-Jump generates test sequences with timing fault detection capabilities. Although, in our case study only timing fault $T F_{B}$ is considered for Controller, other types of timing faults can also be modeled for Controller, Train and Gate processes FUDA03, UWBWF05, UBWF06a. Hitor-Jump can then be used to generate a test sequence which takes into account all of the timing fault models for three processes. Therefore, the test sequences can be used both for unit testing of each process, and for verifying the communication among processes during the integration phase. Another advantage is the flexibility of representing the test sequences in Tree and Tabular Combined Notation (TTCN) [ETSI] or Message Sequence Chart (MSC) [TUZ2 notation, facilitating the portability of the tests.

Table 5. Test sequence generated from SDL specification of Controller

\begin{tabular}{|c|c|c|c|c|c|}
\hline Step No. & Current State & Next State & Cost (Mins.) & Inputs & Outputs \\
\hline 1 & Idle & Sent_Lower & 0 & approach & \\
\hline 2 & Sent_Lower & Sent_Lower & 2 & & \\
\hline 3 & Sent_Lower & Sent_Lower & 0 & & \\
\hline 4 & Sent_Lower & Idle & 0 & & lower \\
\hline 5 & Idle & Sent_Raise & 0 & exit & \\
\hline 6 & Sent_Raise & Sent_Raise & 2 & & \\
\hline 7 & Sent_Raise & Sent_Raise & 0 & & \\
\hline 8 & Sent_Raise & Idle & 0 & & raise \\
\hline
\end{tabular}

\section{Conclusions and Future Work}

In this paper, we apply our timing fault modeling strategy to writing formal specifications for communication protocols. As part of this approach, using the formal language of SDL, we specify the Controller process of rail-road crossing system, a popular benchmark for real-time systems. The EFSM model has the capability of representing a class of timing faults, which otherwise may not be detected in an IUT. We then apply Hit-or-Jump algorithm to the SDL specification based on our EFSM model to generate a test sequence that can detect these timing faults. In addition, including fault modeling into SDL specification 
ensures the synchronization among the timing constraints of different processes, and enables generation of portable test sequences since they can be easily represented in other formal languages such as TTCN or MSC.

As an extension of this work, we will consider the EFSM models with fault detection capabilities for other classes of timing faults, and multiple occurrences of these faults. This approach of modeling the timing faults of communicating processes into formal specifications will also applied to generate integration tests.

\section{References}

[AKLN99] Ashour, M., Khendek, F., Le-Ngoc, T.: Formal description of real-time systems using SDL. In: Proc. of the Sixth Int'l. Conf. on Real-Time Comp. Sys. and Appl (RTCSA'99), Hong-Kong (December 1999)

[ALUR98] Alur, R., Dill, D.: A theory of timed automata. Theoretical Comput. Sci. 126, 183-235 (1994)

[CLRZ99] Cavalli, A., Lee, D., Rinderknecht, C., Zaidi, F.: Hit-or-jump an algorithm for embedded testing with applications to in services. In: Proc. of IFIP Int'l. Conf. FORTE/PSTV'99, (October 1999)

[CMZ04] Cavalli, A., Mederreg, A., Zaidi, F.: Application of a Formal Testing Methodology to Wireless Telephony Networks. Journal of the Brazilian Comp. Soc. 10(2), 56-68 (2004)

[CRV05a] Cavalli, A., Rodrigues, E.: Vieira. Test Case Generation based on Timed Constraints. In: IEEE ICESS 2005, Xian, China (December 2005)

[CRV05b] Cavalli, A., Rodrigues, E.: Vieira. A Formal Approach of Interoperability Test Cases Generation Applied to Real Time Domain. In: IEEE I2TS 05, Florianpolis, SC, Brazil (December 2005)

[DU04] Duale, A.Y., Uyar, M.U.: A method enabling feasible conformance test sequence generation for EFSM models. IEEE Trans. Commun. 53(5), 614-627 (2004)

[EDK02] En-Nouaary, A., Dssouli, R., Khendek, F.: Timed Wp-method: Testing real-time systems. IEEE Trans. Softw. Eng. 28(11), 1023-1038 (2002)

[EDKE98] En-Nouaary, A., Dssouli, R., Khendek, F., Elqortobi, A.: Timed test cases generation based on state characterization technique. In: Proc. IEEE Real-Time Syst. Symp. (RTSS), pp. 220-229, Madrid, Spain (1998)

[EKD99] En-Nouaary, A., Khendek, F., Dssouli, R.: Fault coverage in testing real-time systems. In: Proc. IEEE Int'l Conf. Real-Time Comput. Syst. Appl. (RTCSA), Hong Kong, China (1999)

[ETSI] ETSI. Methods for Testing and Specification (MTS), The Testing and Test Control Notation version 3, Part 1: TTCN-3 Core Language

[FAUD00] Fecko, M.A., Amer, P.D., Uyar, M.U., Duale, A.Y.: Test generation in the presence of conflicting timers. In: Proc. IFIP Int'l Conf. Test. Commun. Syst. (TestCom), pp. 301-320, Ottawa, Canada (2000)

[FUDA03] Fecko, M.A., Uyar, M.U., Duale, A.Y., Amer, P.D.: A technique to generate feasible tests for communications systems with multiple timers. IEEE/ACM Trans. Netw. 11(5), 796-809 (2003)

[HJL93] Heitmeyer, C.L., Jeffords, R.D., Labaw, B.G., Benchmark, A.: for Comparing Different Approaches for Specifying and Verifying Real-Time Systems. In: Proc. Tenth Int'l. Workshop on Real-Time Operating Sys. and Software (May 1993) 
[HL94] Heitmeyer, C., Lynch, N.: The Generalized Railroad Crossing: A Case Study in Formal Verification of Real-Time System. In: Proc. of the 15th IEEE Real-Time Sys. Symp., Puerto Rico (December 1994)

[HL96] Heitmeyer, C., Lynch, N.: Formal Verification of Real-time Systems Using Timed Automata. In: Heitmeyer, C., Lynch, N. (eds.) Trends in Formal Methods for Real-Time Computing, pp. 83-106. John Wiley and Sons, Ltd, Chichester (1996)

[ITUZ1] ITU-T. Rec. Z.100 Specification and Description Language (1980)

[ITUZ2] ITU-T. Rec. Z. 120 Message Sequence Charts, Geneva (1996)

[LRS98] Lanphier, R., Rao, A., Schulzrinne, H.: Real time streaming protocol (RTSP). RFC 2326, IETF (1998)

[SCFJ96] Schulzrinne, H., Casner, S., Frederick, R., Jacobson, V.: RTP: A transport protocol for real-time applications. RFC 1889, IETF (1996)

[TMCB03] Teyssie, C., Mmammeri, Z., Carcenac, F., Buniol, F.: Etude Comparative de SDL et UML pour la Modelisation de Systemes Temps Reel. In: 11th Conf. on Real-Time and Embedded Systems, pp. 75-97, Paris, Teknea (April 2003)

[UWBWF05] Uyar, M.U., Wang, Y., Batth, S.S., Wise, A., Fecko, M.A.: Timing Fault Models for Systems with Multiple Timers, IFIP Int'l. Conf. on Testing of Comm. Systems (TESTCOM), Concordia, Canada (2005)

[UBWF06a] Uyar, M.U., Batth, S.S., Wang, Y., Fecko, M.A.: EFSM graph augmentation algorithms for modeling a class of single timing faults. IEEE Trans. Comput. (In review 2006)

[UfDA01] Uyar, M.U., Fecko, M.A., Duale, A.Y., Amer, P.D., Sethi, A.S.: A formal approach to development of network protocols: Theory and application to a wireless standard. In: Proc. Concordia Prestigious Wksp Commun. Softw. Eng. (CPWCSE), Montreal, Canada (invited paper) (2001)

[UZ93] Ural, H., Zhu, K.: Optimal length test sequence generation using distinguishing sequences. IEEE/ACM Trans. Netw. 1(3), 358-371 (1993)

[XEN04] Xiang, Z., En-Nouaary, A.: Test cases generation for embedded realtime systems based on test purposes. In: NOTERE'2004, Saidia, Maroc (Juin 2004) 